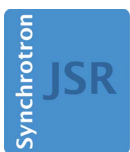

JOURNAL OF SYNCHROTRON RADIATION

ISSN 1600-5775

Received 24 November 2014

Accepted 3 March 2015

Edited by M. Yabashi, RIKEN SPring-8 Center, Japan

\# Currently at Korea Research Institute of Standards and Science, Daejeon 305-600, Korea.

Keywords: FEL; hard X-ray; XPCS; coherent scattering.

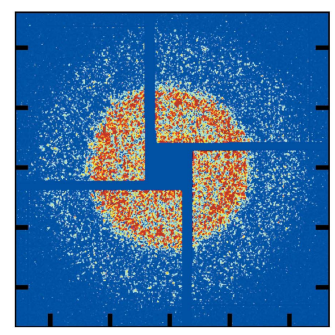

OPEN $\odot$ ACCESS

\section{The X-ray Correlation Spectroscopy instrument at the Linac Coherent Light Source}

\author{
Roberto Alonso-Mori, ${ }^{a}$ Chiara Caronna, ${ }^{a}$ Matthieu Chollet, ${ }^{a}$ Robin Curtis, \\ Daniel S. Damiani, ${ }^{a}$ Jim Defever, ${ }^{a}$ Yiping Feng, ${ }^{a}$ Daniel L. Flath, ${ }^{\text {a }}$ \\ James M. Glownia, ${ }^{a}$ Sooheyong Lee, ${ }^{a, b} \neq$ Henrik T. Lemke, ${ }^{a}$ Silke Nelson, ${ }^{a}$ \\ Eric Bong, ${ }^{a}$ Marcin Sikorski, ${ }^{a}$ Sanghoon Song, ${ }^{a}$ Venkat Srinivasan, ${ }^{a}$ \\ Daniel Stefanescu, ${ }^{a}$ Diling $\mathrm{Zhu}^{\mathrm{a}}$ and Aymeric Robert ${ }^{\mathrm{a} *}$
}

a Linac Coherent Light Source, SLAC National Accelerator Laboratory, 2575 Sand Hill Road, Menlo Park,
CA 94025, USA, and ${ }^{\text {b}}$ Hasylab at DESY, Notkestrasse 85, D-22607 Hamburg, Germany.
${ }^{*}$ Correspondence e-mail: aymeric@slac.stanford.edu

The X-ray Correlation Spectroscopy instrument is dedicated to the study of dynamics in condensed matter systems using the unique coherence properties of free-electron lasers. It covers a photon energy range of $4-25 \mathrm{keV}$. The intrinsic temporal characteristics of the Linac Coherent Light Source, in particular the $120 \mathrm{~Hz}$ repetition rate, allow for the investigation of slow dynamics (milliseconds) by means of X-ray photon correlation spectroscopy. Double-pulse schemes could probe dynamics on the picosecond timescale. A description of the instrument capabilities and recent achievements is presented.

\section{Introduction}

The Linac Coherent Light Source (LCLS), a US Department of Energy Office of Science user facility operated by Stanford University, achieved first light in 2009 (Emma et al., 2010). The X-ray Correlation Spectroscopy (XCS) instrument began operations in 2011; located in the Far Experimental Hall (FEH), XCS was the fifth LCLS instrument to become operational. In contrast with synchrotron storage ring sources, which provide high-brilliance beam with partial coherence (Grübel et al., 2008), LCLS provides pulsed transversely coherent hard X-rays with unprecedented flux and short pulse duration.

These characteristics enable the investigation of dynamics in condensed matter by measuring time-resolved coherent scattering patterns (i.e. time-resolved speckles). This can be achieved by means of X-ray Photon Correlation Spectroscopy (XPCS) (Grübel et al., 2008), where the temporal evolution of speckle patterns can be quantified by calculating intensity autocorrelation functions and extracting typical relaxation times for specific length scales (Robert, 2007). The LCLS repetition rate, $120 \mathrm{~Hz}$, limits the fastest measurable time scales to several tens of milliseconds (Stephenson et al., 2009).

More elaborate schemes, requiring the measurement of the sum of two speckle patterns originating from a sequence of two X-ray pulses separated in time (Gutt et al., 2009), allow the measurement of much faster dynamics. In that case the accessible time scales are limited by the ability to generate two pulses separated in time by $\Delta t$ and can potentially reach the femtosecond regime.

The XCS instrument was designed to provide an optimum platform for taking advantage of the unique coherence properties of the LCLS (e.g. the possibility to access very large 
sample-detector distances) while still accommodating experiments using different X-ray techniques (small-angle $\mathrm{X}$-ray scattering, diffraction, X-ray spectroscopy and imaging) (Robert et al., 2013). The addition of an ultrafast laser system will not only allow 'standard' optical pump/X-ray probe experiments but will open new opportunities to use coherent scattering techniques in combination with ultrafast optical excitation.

\section{Instrument overview}

The XCS instrument operates in the hard X-ray regime (above $4 \mathrm{keV}$ ). A set of silicon mirrors (coated with silicon carbide), with an incidence angle of $1.32 \mathrm{mrad}$, are located in the frontend enclosure of the Near Experimental Hall (NEH), which feeds the hard X-ray beam to all LCLS hard X-ray hutches. These limit the maximum photon energy that can be delivered to the LCLS hard X-ray hutches to $25 \mathrm{keV}$.

The first components of the XCS instrument are located in the X-ray Transport Tunnel, a $200 \mathrm{~m}$-long tunnel connecting the Near and Far Experimental Halls. These include slits and diagnostics located $196 \mathrm{~m}$ upstream from the sample, as indicated in Fig. 1.

In the following, we describe the specifications of key components of the XCS instrument.

Monochromators. The XCS instrument has two distinct configurations. It can operate in the LCLS main line and take full advantage of the first-harmonic properties of LCLS and its various operation modes (SASE, two-color, etc.). To operate in this 'pink beam' configuration, all components located downstream of the dashed line in Fig. 1 are translated to the LCLS main line. For experiments that require a monochromatic beam [i.e. a larger longitudinal coherence length $\xi_{1}=$ $\left.\lambda^{2} / \Delta \lambda=\lambda(E / \Delta E)\right]$ but do not intend to scan broadly the incident energy, a custom-built (JJ X-ray, Denmark) largeoffset Double-Crystal Monochromator (DCM) (Zhu et al.,

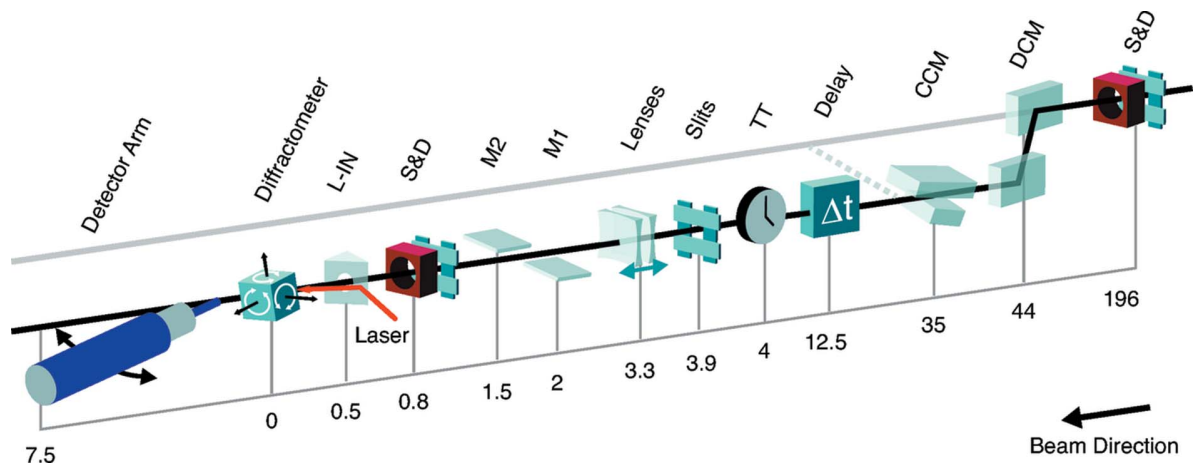

Figure 1

Overview of the XCS instrument layout. Distances are indicated in meters from the center of the diffractometer. S\&D: slits and non-destructive intensity diagnostics; DCM: large-offset doublecrystal monochromator; CCM: channel-cut monochromator; TT: time-tool measuring the arrival time of the optical laser with reference to the X-rays; M1/M2: silicon mirrors that can be used to deflect the beam in the vertical direction and can also provide harmonic rejection; L-IN: laser incoupling for the optical laser. Components located downstream of the dashed line can be translated into the main LCLS line and allow the XCS instrument to take advantage of the full power and properties of the fundamental. The sample at the XCS instrument is located approximately $420 \mathrm{~m}$ from the source.
2014 ) is located $44 \mathrm{~m}$ upstream of the sample. In this configuration all components located downstream of the dashed line are translated $600 \mathrm{~mm}$ horizontally as displayed in Fig. 1. The DCM operates with $\mathrm{Si}(111)$ crystals and provides a monochromaticity of $\Delta E / E=1.4 \times 10^{-4}$ at $8 \mathrm{keV}$. If more monochromaticity is required, a $\mathrm{Si}(511)$ Channel-Cut Monochromator (CCM) (Narayanan et al., 2008) operating in the vertical scattering geometry provides an energy resolution of $\Delta E / E=8.9 \times 10^{-6}$. Future plans include an upgrade of the DCM crystals to $C^{*}(111)$, which not only provides better energy resolution $\left(\Delta E / E=5.3 \times 10^{-5}\right)$ but most importantly allows multiplexing with another instrument located downstream in the FEH (Feng et al., 2013, 2015; Zhu et al., 2014). A detailed discussion concerning the effect of the monochromaticity on the intensity and longitudinal coherence properties of a FEL SASE beam is provided by Lee et al. (2012).

$X$-ray focusing lenses. The unfocused beam at XCS is typically $0.75 \mathrm{~mm} \times 0.75 \mathrm{~mm} \mathrm{FWHM} \mathrm{in} \mathrm{size.} \mathrm{Beryllium}$ compound refractive lenses (RWTH, Aachen, Germany) allow focusing of the beam, and provide beam size control in one or two dimensions (Snigirev et al., 1996). The lenses are located $3.3 \mathrm{~m}$ upstream of the sample and can be adjusted $\pm 150 \mathrm{~mm}$ along the incident beam axis. The minimum spot size was measured to be $\sim 2 \mu \mathrm{m} \times 2 \mu \mathrm{m}$ (limited by the finite $\mathrm{SE}$ bandwidth and imperfections in the optics). A oneensional focusing option allows delivery of a line focus for dence experiments.

Pulse picker. A fast shutter is available for selecting single $\mathrm{X}$-ray pulses from the LCLS pulse train on demand, as well as for reducing the repetition rate. It consists of a channel which is rotated back and forth in order to create a brief opening time. It can be used to create arbitrary pulse train time patterns provided that the pulse train structure has an average rate of equal to or less than $10 \mathrm{~Hz}$.

Mirrors. Two silicon mirrors located 1.5 and $2 \mathrm{~m}$ upstream of the sample allow delivery of the beam with a vertical grazing angle (as, for example, is required for liquid surfaces). These can also reduce the third-harmonic content of the LCLS beam by operating above the critical angle for these energies.

Diffractometer. A custom-built horizontal geometry four-circle diffractometer (Huber, Germany) is available and enables precise orientation of samples or sample environments such as vacuum chambers, gas/liquid injectors, etc. Its sphere of confusion is better than $20 \mu \mathrm{m}$. It can be used in conjunction with the auxiliary $2 \theta$ arm (FMB Oxford, UK) providing large sample-detector distances (4 and $7.5 \mathrm{~m}$ ) and covering scattering angles up to $55^{\circ}$ in the horizontal plane. 
Additional diagnostics. The SASE process induces pulse-topulse fluctuations of the beam properties, such as pulse energy, duration, spatial profile, wavefront, temporal profile and spectral content. In situ pulse property monitoring is thus crucial for data interpretation. Multiple intensity monitors are installed at various locations along the instrument for pulseto-pulse intensity normalization (Feng et al., 2011). The spatial profile of the beam can also be measured at various locations along the instrument using scintillating screens with highresolution camera-lens combinations.

Detectors. Several X-ray detectors are available and integrated with the XCS data acquisition system. These have various characteristics (pixel size, number of pixel, noise, frame rate and dynamic range) which are evaluated in order to identify the most suitable detector for specific experimental needs. Coherent X-ray experiments, for example, typically require small pixel size, very low noise, moderate dynamic range and a large number of pixels. This can be achieved with the $20 \mu \mathrm{m}$ pixel size direct illumination Princeton CDD, but at a very low frame rate. A new detector is currently being developed at LCLS with $55 \mu \mathrm{m}$ pixels and low noise, but running at the $120 \mathrm{~Hz}$ full repetition rate (Dragone et al., 2014). For more information about the LCLS detectors, see Blaj et al. (2015).

Split and delay. The XCS instrument has space allocated for instrumentation to generate double-pulse X-ray patterns with a controlled $\Delta t$ typically below $1 \mathrm{~ns}$. A split and delay prototype built by DESY (Hamburg, Germany) is currently installed and its performances are described elsewhere (Roseker et al., 2009, 2012). Other prototypes offering different beam properties are being evaluated or tested.

Ultrafast laser capabilities will be added to the XCS instrument in 2015. These include the construction of a dedicated laser hutch in close proximity to XCS, a standard ultrafast laser system, and timing diagnostics; the specifications of each are listed below:

Optical laser system. Core laser systems at the LCLS consist of an ultrashort-pulse Ti:sapphire oscillator synchronized to the FEL seeding a commercially available chirped pulse amplifier producing $4 \mathrm{~mJ}$ at $40 \mathrm{fs}$. An additional four-pass amplifier, developed in-house, can boost the pulse energy to over $30 \mathrm{~mJ}$. Wavelength conversion can cover a broad spectral range from $200 \mathrm{~nm}$ to $150 \mu \mathrm{m}$ ( $\sim 1500$ to $2 \mathrm{THz})$. A more thorough description of the optical laser capabilities at LCLS can be found by Minitti et al. (2015).

Timing diagnostics. Typical phase locking between the accelerator and the laser system only holds the temporal jitter between the two sources to about 200 fs FWHM. In order to take full advantage of the short pulses and reach pulse-lengthlimited time resolution, diagnostics to measure the relative arrival time between laser and X-ray pulses have been developed. These are based on the X-ray induced change in refractive index of a thin target probed by a chirped broadband white-light continuum pulse derived from the optical laser. The optical light transmission change is resolved by an optical spectrometer for each pulse. Typical target materials are silicon nitride $\left(\mathrm{Si}_{3} \mathrm{~N}_{4}\right)$ or Ce:YAG crystals of different
Table 1

X-ray parameters and capabilities of the XCS instrument.

\begin{tabular}{ll}
\hline Instrument name & XCS \\
Mirrors, incidence angle & $2 \mathrm{SiC}$ on Si, $1.32 \mathrm{mrad}$ \\
Monochromaticity $(\Delta E / E) \dagger$ & $1 \times 10^{-3}$ (SASE), $2 \times 10^{-4}$ (seeding) \\
Energy range $(\mathrm{keV})$ & 4 to 11 (fundamental) \\
Unfocused beam size $(\mu \mathrm{m})$ & 750 at $8.3 \mathrm{keV}$ \\
Focused beam size $(\mu \mathrm{m})$ & $2-750$ \\
Focusing optics & Be lenses, 1D and 2D focusing \\
Flux (photons pulse $\left.{ }^{-1}\right)$ & $1 \times 10^{12}$ (fundamental) $\ddagger$ \\
Pulse length $(\mathrm{fs})$ & $5-200$ \\
Repetition rate $(\mathrm{Hz})$ & $120,60,30,10,5,1$, on demand \\
Optical laser pulse energy $(\mathrm{mJ}) \S$ & $20(800 \mathrm{~nm}), 4-5(400 \mathrm{~nm}), 1(266 \mathrm{~nm})$ \\
Optical laser pulse length $(\mathrm{fs}) \S$ & $10-150$ \\
Standard detectors & CSPAD, CSPAD-140k, ePix \\
& Princeton \\
Sample environment & Huber horizontal four-circle \\
& diffractometer, general purpose \\
& vacuum, liquid jet, He enclosure, \\
& Oxford LN $\mathrm{N}_{2}$ cryojet down to $100 \mathrm{~K}$ \\
$2 \theta$ arm capabilities & Large sample-detector distance arm \\
& $(4$ and $7.5 \mathrm{~m})$ covering $0^{\circ}<2 \theta<55^{\circ}$ \\
\hline
\end{tabular}

$\dagger$ Typical single-shot value. $\quad$ Excluding beamline and instrument transmission. $\S$ To be installed in 2015 .

thicknesses to accommodate different beam conditions (Lemke et al., 2013).

A summary of the XCS instrument parameters is given in Table 1.

\section{Highlights}

The XCS instrument focuses on measuring time-resolved coherent scattering patterns from condensed matter systems from which typical relaxation rates are deduced. These measurements take full advantage of the transverse coherence properties of the LCLS beam. Fig. 2 displays a typical singleshot speckle pattern from a static sample consisting of dried silica colloidal $150 \mathrm{~nm}$ diameter spheres (Kisker $\mathrm{GmbH}$ ) with $8.3 \mathrm{keV}$ X-rays. The detector is a CSPAD (Blaj et al., 2015) located $7.5 \mathrm{~m}$ downstream of the sample. The concentric excess scattering signals observed at $Q \simeq 0.005 \AA^{-1}$ and $Q \simeq$

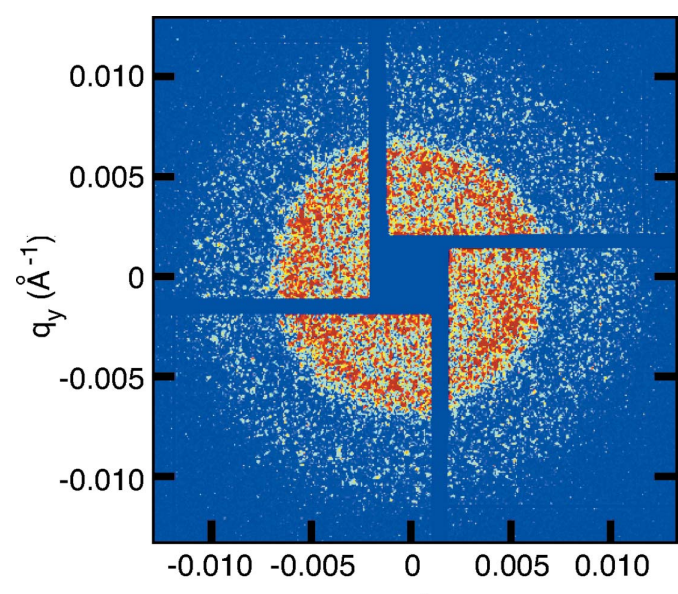

Figure $2 \quad \mathrm{q}_{\mathrm{x}}\left(\AA^{-1}\right)$

Single-shot speckle pattern measured at $8.3 \mathrm{keV}$ from $150 \mathrm{~nm}$ colloidal spheres. The dark blue areas are gaps between the CSPAD tiled sensors. The central aperture allows the transmitted beam to pass through, and therefore does not require a beamstop. 


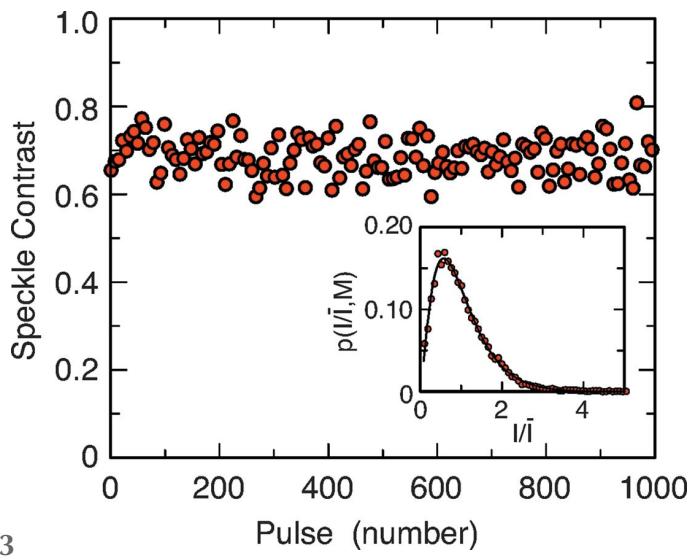

Figure 3

Single-shot speckle contrast measured at $Q=0.0067 \AA^{-1}$ for various consecutive shots. Inset: probability density function of intensity within part of the speckle pattern corresponding to a wavevector $Q=$ $0.0067 \AA^{-1}$. The solid line represents the gamma distribution with number of modes $M=2.75$ and average count rate $\bar{I} \simeq 5.1$ photons.

$0.01 \AA^{-1}$ are typical small-angle scattering form-factor features related to the size and shape of the colloidal particles. As observed, the speckles (coherent scattering pattern appearing as the grainy features decorating the structure rings) are well developed.

In order to characterize the transverse coherence of the beam, a series of speckle patterns are measured with and without the XCS monochromator, in the SASE operation mode, and analyzed in terms of photon statistics to determine their contrast, i.e. a measure of the transverse coherence in the small-angle regime (Gutt et al., 2012; Hruszkewycz et al., 2012; Lee et al., 2013; Lehmkühler et al., 2014). The intensities from a narrow iso- $Q$ area consisting of an annulus centered at $Q=0$ with a radius $Q$ are histogrammed as displayed in Fig. 3 for $Q=0.0067 \AA^{-1}$. These are then modeled using the negativebinomial distribution function (Mandel, 1959; Goodman, 2007)

$$
P_{M}(I)=\frac{\Gamma(I+M)}{\Gamma(M) \Gamma(I+1)}\left(1+\frac{M}{\bar{I}}\right)^{-I}\left(1+\frac{\bar{I}}{M}\right)^{-M}
$$

where $I$ is the number of photons, $\bar{I}$ is the mean number of photons in that area and $M$ is the number of modes. $M$ is related to the contrast of the speckle pattern $C=1 / \sqrt{M}$. An example of the result of such analysis is displayed in Fig. 3 (inset). For that specific shot the fit to the experimental data yields a mean number $\bar{I} \simeq 5.1$ photons, mode number $M=2.75$ corresponding to a contrast $C=0.6$. The fit reproduces well the experimentally measured single-shot intensity distribution.

Because the mean number of photons is large one can also use a simpler formulation of the speckle contrast $C=\sigma / \bar{I}$, where $\sigma$ is the standard deviation of the measured intensities. This analysis was performed on successive shots at the same $Q=0.0067 \AA^{-1}$. The results are displayed in Fig. 3. The observed contrast fluctuates between 0.6 and 0.8 with a mean contrast of 0.69 . The shot-to-shot fluctuations of the contrast originate from the fine structures in the energy spectrum of the SASE beam, as simulated and described by Lee et al. (2012).
The large degree of coherence of the LCLS beam makes it a suitable source to perform XPCS, measuring time-resolved speckle patterns from a sample. As the sample presents some dynamics, speckle patterns fluctuate in time. By means of timeautocorrelation functions calculated from the speckle patterns, information on the characteristic relaxation times can be obtained (Robert, 2007), a measure of the normalized intermediate scattering function of the system. In some cases the sample can also undergo non-equilibrium dynamical phenomena, referred to as aging (Robert et al., 2006), from which clear signatures can be observed in a time-dependent analysis of the degree of correlation of speckle patterns. This was recently investigated at XCS by Carnis et al. (2014), where the relaxation and aging dynamics of thin polymer films were investigated. One should also note that with a FEL the spatial position of the beam jitters shot-to-shot. Because of this effect the contrast $\beta$, usually referred to in second-order correlation functions (Robert, 2007), will be smaller than the single-shot speckle contrast, as discussed by Carnis et al. (2014).

Time-resolved speckle spectroscopy at an FEL is intrinsically limited by the repetition rate of the source, as it relies on the time-correlation of two recorded coherently scattered patterns originating from two pulses separated in time. For the LCLS, the fastest dynamics that can be reached is of the order of $8.3 \mathrm{~ms}$ (i.e. $120 \mathrm{~Hz}$ repetition rate). A natural way of accessing faster timescales is to increase the repetition rate of the source. This would require a completely different accelerator technology such as the one planned to be used at the European XFEL (Grübel et al., 2007), where series of X-ray bunches separated by a minimum of $220 \mathrm{~ns}$ will be generated.

To reach even faster timescales down to the picosecond regime and below, other possibilities involve generating two sub-pulses with a time separation $\Delta t$ between tens of femtoseconds up to a nanosecond as illustrated in Fig. 4. Each subpulse generates a speckle pattern. Current X-ray detectors are, however, only capable of measuring the sum of the scattered signal of both sub-pulses. If dynamics occurs on timescales of $\Delta t$, the summed speckle patterns will present a decay of contrast, as compared with the contrast of a single speckle pattern. By adjusting the time delay $\Delta t$ between the two subpulses, one can extract the time evolution of the summed speckle pattern contrast, from which the normalized intermediate scattering function is obtained (Gutt et al., 2009), and therefore gain information about the underlying dynamics of the system.

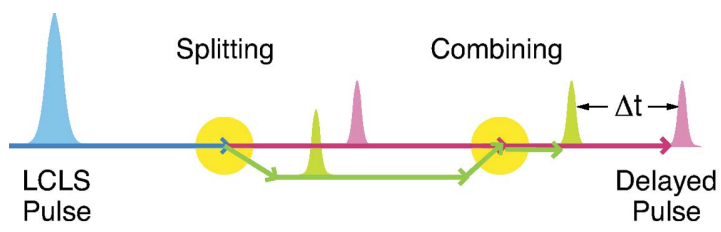

Figure 4

Schematic of the double-pulse scheme. A single pulse is split into two subpulses; one is delayed relative to the other by an increase in its pathlength. The sub-pulses, separated in time by $\Delta t$, are then redirected on a common trajectory to the sample. A typical pathlength difference of $1 \mathrm{~mm}$ corresponds to a time delay of $3 \mathrm{ps}$. 
Recent results from the commissioning of a split and delay prototype (Roseker et al., 2012) based on eight perfect Bragg crystals [including two thin crystals that can act as a beam splitter/recombiner (Roseker et al., 2009)] have shown the possibility to reach time delays of the order of a nanosecond.

Other technical developments involving split and delayed beams have also been investigated. This is particularly the case with a single-shot split and multiple-delay system, offering the possibility to probe ultrafast dynamics by means of X-ray pump/X-ray probe experiments, as recently demonstrated by David et al. (2015).

\section{Conclusion}

The XCS instrument takes full advantage of the large number of transversely coherent photons per pulse at the LCLS. XCS is a versatile tool for performing time-resolved coherent scattering experiments in the hard X-ray regime from which fundamental dynamics in condensed matter ordered and disordered systems can be explored. This can be achieved by means of XPCS for slow dynamics and will further be extended to ultrafast timescales by double-pulse experiments. More details about the XCS instrument can be found on the following website: http://lcls.slac.stanford.edu/xcs.

\section{Facility access}

LCLS instruments are open to academia, industry, government agencies and research institutes worldwide for scientific investigations. There are two calls for proposals per year and an external peer-review committee evaluates proposals based on scientific merit and instrument suitability. Access is without charge for users who intend to publish their results. Prospective users are encouraged to contact instrument staff members to learn more about the science and capabilities of the facility, and opportunities for collaboration.

\section{Acknowledgements}

We thank Terry Anderson and Gregory Stewart for helping with the figures. Portions of this research were carried out at the Linac Coherent Light Source (LCLS) at the SLAC National Accelerator Laboratory. LCLS is an Office of Science User Facility operated for the US Department of Energy Office of Science by Stanford University.

\section{References}

Blaj, G., Caragiulo, P., Carini, G., Carron, S., Dragone, A., Freitag, D., Haller, G., Hart, P., Hasi, J., Herbst, R., Herrmann, S., Kenney, C., Markovic, B., Nishimura, K., Osier, S., Pines, J., Reese, B., Segal, J., Tomada, A. \& Weaver, M. (2015). J. Synchrotron Rad. 22, 577-583.

Carnis, J., Cha, W., Wingert, J., Kang, J., Jiang, Z., Song, S., Sikorski, M., Robert, A., Gutt, C., Chen, S. W., Dai, Y., Ma, Y., Guo, H. B. L. L., Shpyrko, O., Narayanan, S., Cui, M., Kosif, I., Emrick, T., Russell, T. P., Lee, H. C., Yu, C., Grübel, G., Sinha, S. K. \& Kim, H. (2014). Sci. Rep. 4, 6017.

David, C., Karvinen, P., Sikorski, M., Song, S., Vartiainen, I., Milne, C. J., Mozzanica, A., Kayser, Y., Diaz, A., Mohacsi, I., Carini, G. A.,
Herrmann, S., Färm, E., Ritala, M., Fritz, D. M. \& Robert, A. (2015). Sci. Rep. 5, 7644.

Dragone, A., Caragiulo, P., Markovic, B., Herbst, R., Reese, B., Herrmann, S. C., Hart, P. A., Segal, J., Carini, G. A., Kenney, C. \& Haller, G. (2014). J. Phys. Conf. Ser. 493, 012012.

Emma, P. et al. (2010). Nat. Photon. 4, 641-647.

Feng, Y., Alonso-Mori, R., Barends, T., Blank, V., Botha, S., Chollet, M., Damiani, D. S., Doak, R. B., Glownia, J. M., Koglin, J. M., Lemke, H. T., Messerschmidt, M., Nass, K., Nelson, S., Schlichting, I., Shoeman, R. L., Shvyd'ko, Y. V., Sikorski, M., Song, S., Stoupin, S., Terentyev, S., Williams, G. J., Zhu, D., Robert, A. \& Boutet, S. (2015). J. Synchrotron Rad. 22, 626-633.

Feng, Y., Alonso-Mori, R., Blank, V., Boutet, S., Chollet, M., van Driel, T. B., Fritz, D. M., Glownia, J. M., Hastings, J. B., Lemke, H., Messerchmidt, M., Montanez, P. A., Robert, A., Robinson, J., Samoylova, L., Shvyd'ko, Y., Sikorski, M., Sinn, H., Song, S., Srinivasan, V. N., Stoupin, S., Terentiev, S., Williams, G. \& Zhu, D. (2013). Proc. SPIE, 8778, 87780B.

Feng, Y., Feldkamp, J. M., Fritz, D. M., Cammarata, M., Aymeric, R., Caronna, C., Lemke, H. T., Zhu, D., Lee, S., Boutet, S., Williams, G., Tono, K., Yabashi, M. \& Hastings, J. B. (2011). Proc. SPIE, 8140, 81400Q.

Goodman, J. W. (2007). Speckle Phenomena in Optics: Theory and Applications. Roberts and Company.

Grübel, G., Madsen, A. \& Robert, A. (2008). Soft Matter Characterization, edited by R. Borsali and R. Pecora, ch. 18, pp. 953-995. Berlin: Springer.

Grübel, G., Stephenson, G. B., Gutt, C., Sinn, H. \& Tschentscher, T. (2007). Nucl. Instrum. Methods Phys. Res. B, 262, 357-367.

Gutt, C., Stadler, L.-M., Duri, A., Autenrieth, T., Leupold, O., Chushkin, Y. \& Grübel, G. (2009). Opt. Express, 17, 55-61.

Gutt, C., Wochner, P., Fischer, B., Conrad, H., Castro-Colin, M., Lee, S., Lehmkühler, F., Steinke, I., Sprung, M., Roseker, W., Zhu, D., Lemke, H., Bogle, S., Fuoss, P. H., Stephenson, G. B., Cammarata, M., Fritz, D. M., Robert, A. \& Grübel, G. (2012). Phys. Rev. Lett. 108, 024801.

Hruszkewycz, S. O., Sutton, M., Fuoss, P. H., Adams, B., Rosenkranz, S., Ludwig, K. F., Roseker, W., Fritz, D., Cammarata, M., Zhu, D., Lee, S., Lemke, H., Gutt, C., Robert, A., Grübel, G. \& Stephenson, G. B. (2012). Phys. Rev. Lett. 109, 185502.

Lee, S., Roseker, W., Gutt, C., Fischer, B., Conrad, H., Lehmkühler, F., Steinke, I., Zhu, D., Lemke, H., Cammarata, M., Fritz, D. M., Wochner, P., Castro-Colin, M., Hruszkewycz, S. O., Fuoss, P. H., Stephenson, G. B., Grübel, G. \& Robert, A. (2013). Opt. Express, 21, 24647-24664.

Lee, S., Roseker, W., Gutt, C., Huang, Z., Ding, Y., Grübel, G. \& Robert, A. (2012). Opt. Express, 20, 9790-9800.

Lehmkühler, F., Gutt, C., Fischer, B., Schroer, M. A., Sikorski, M., Song, S., Roseker, W., Glownia, J., Chollet, M., Nelson, S., Tono, K., Katayama, T., Yabashi, M., Ishikawa, T., Robert, A. \& Grübel, G. (2014). Sci. Rep. 4, 5234.

Lemke, H. T., Weaver, M., Chollet, M., Robinson, J., Glownia, J. M., Zhu, D., Bionta, M. R., Cammarata, M., Harmand, M., Coffee, R. N. \& Fritz, D. M. (2013). Proc. SPIE, 8778, 87780S.

Mandel, L. (1959). Proc. Phys. Soc. 74, 233.

Minitti, M. P., Robinson, J. S., Coffee, R. N., Edstrom, S., Gilevich, S., Glownia, J. M., Granados, E., Hering, P., Hoffmann, M. C., Miahnahri, A., Milathianaki, D., Polzin, W., Ratner, D., Tavella, F., Vetter, S., Welch, M., White, W. E. \& Fry, A. R. (2015). J. Synchrotron Rad. 22, 526-531.

Narayanan, S., Sandy, A., Shu, D., Sprung, M., Preissner, C. \& Sullivan, J. (2008). J. Synchrotron Rad. 15, 12-18.

Robert, A. (2007). J. Appl. Cryst. 40, s34-s37.

Robert, A., Curtis, R., Flath, D., Gray, A., Sikorski, M., Song, S., Srinivasan, V. \& Stefanescu, D. (2013). J. Phys. Conf. Ser. 425, 212009.

Robert, A., Wandersman, E., Dubois, E., Dupuis, V. \& Perzynski, R. (2006). Europhys. Lett. 75, 764-770. 
Roseker, W., Franz, H., Schulte-Schrepping, H., Ehnes, A., Leupold, O., Zontone, F., Robert, A. \& Grübel, G. (2009). Opt. Lett. 34, 1768-1770.

Roseker, W., Lee, S., Walther, M., Schulte-Schrepping, H., Franz, H., Gray, A., Sikorski, M., Fuoss, P. H., Stephenson, G. B., Robert, A. \& Grübel, G. (2012). Proc. SPIE, 8504, 85040I.

Snigirev, A., Kohn, V., Snigireva, I. \& Lengeler, B. (1996). Nature (London), 384, 49-51.
Stephenson, G. B., Robert, A. \& Grübel, G. (2009). Nat. Mater. 8, 702-703.

Zhu, D., Feng, Y., Stoupin, S., Terentyev, S. A., Lemke, H. T., Fritz, D. M., Chollet, M., Glownia, J. M., Alonso-Mori, R., Sikorski, M., Song, S., van Driel, T. B., Williams, G. J., Messerschmidt, M., Boutet, S., Blank, V. D., Shvyd'ko, Y. V. \& Robert, A. (2014). Rev. Sci. Instrum. 85, 063106. 